\title{
Viewpoint: Adult infective encephalitis early diagnosis and treatment is imperative
}

\section{Bakış Açısı: Yetişkin enfektif ensefaliti erken teşhis ve tedavi şarttır}

\author{
Husayn Al Mahdy ${ }^{1}$
}

1 Department of Medicine Yeovil District Hospital Yeovil -United Kingdom

\begin{abstract}
Purpose: Adult infective encephalitis tends to be managed inadequately largely due to investigative modalities that are non -specific in the early disease process. It is therefore critical that new blood and cerebrospinal fluid tests be added to the list of investigations that can lead to a diagnosis of infective encephalitis early, as delay increases morbidity and mortality.
\end{abstract}

Key words: Infective encephalitis, diagnosis, treatment 


\section{Introduction}

The clinical literature over the past decades consisted of numerous articles on diagnosis and treatment of adult infective encephalitis giving the impression that it is an easy process. Clinical experience dictates that the diagnosis can be exceptionally difficult leading to delay in treatment. This brief article attempts to show why this is so and provides avenues where further study is required in expediting the diagnosis and therefore treatment.

\section{Terminology}

Encephalitis denotes inflammation of the brain substance leading to neurological symptoms and signs. When the inflammatory process involves the contiguous meninges the term meningo-encephalitis applies. On occasions an encephalitic process is established from a previous vaccination in an immunocompetent person which is a recognised clinical entity and this is termed post immunisation encephalitis. In this situation no infective organism is involved and it is the immune response of the patient to the vaccine component that produces such clinical picture. Autoimmune encephalitis is another entity that needs to be borne in mind when a diagnosis of encephalitis is entertained. This brief article will only deal with infective encephalitis as the other forms of encephalitis necessitate their own extensive study.

Clinical presentation of infective encephalitis

The clinical presentation is usually rapid with fever, confusion, drowsiness and sometimes seizures as well as focal neurological signs depending on the area of the brain most involved. It is not uncommon for an infection affecting a remote part of the body such as urinary tract infection in older people to present as confusion and pyrexia without accompanying encephalitis and such a presentation may confuse the clinical picture.
Similarly, metabolic derangement may also present with confusion especially those with decompensated cirrhosis without encephalitis; and all such presentations are termed encephalopathy. Initially such distinction can be difficult but within a reasonable time frame coupled with clinical experience taking into account the clinical picture, the working diagnosis becomes clearer. This is particularly the case in elderly patients who may present with urinary tract infection, particularly pyelonephritis, manifesting with confusion and pyrexia when the pre-morbid state was reasonable. In such cases the organ involved will make itself apparent by presence of cloudy and smelly urine. Those who present with pneumonia leading to confusion and headache, the additional clinical features of productive cough of green/grey phlegm, hypoxia and tachypnoenia with chest signs on auscultation and supported by chest $\mathrm{x}$-ray will settle the issue of encephalopathy or encephalitis.

Occasionally infection at a different part of the body such as mycoplasma pneumonia or pulmonary tuberculosis may have accompanying encephalitis. Hence a search for such of focus of infection may make the diagnosis and management of associated encephalitis easier coupled with relevant tissue/body secretions culture, imaging of the brain and cerebrospinal fluid (CSF) examination. It is vital for rapid and accurate diagnosis of infective encephalitis coupled with expeditious management, that a comprehensive history including travel, sexual, occupational and animal exposure together with a thorough clinical examination, supported by relevant investigations are undertaken.

The micro-organisms commonly producing infective encephalitis in immunocompetent adults are Herpes simplex (Type I and less commonly Type II) viruses, Herpes zoster and 
Enteroviruses (Table 1) [1]. Other viruses encountered in immunocompetent individuals are confined to specific geographical areas, like West Nile Virus, Japanese Encephalitis virus, and St. Louise virus.

Table 1. Micro-organisms in encephalitis

\begin{tabular}{|l|l|l|}
\hline Viruses* & $\begin{array}{l}\text { Herpes simplex Types I \& } \\
\text { II Herpes zoster } \\
\text { Enteroviruses } \\
\text { Cytomegalovirus } \\
\text { Epstein-Barr virus }\end{array}$ & $\begin{array}{l}\text { usually in } \\
\text { immunocompromised J } \\
\text { individuals }\end{array}$ \\
\hline Bacteria & $\begin{array}{l}\text { Mycoplasma pneumonia } \\
\text { Listeria monocygenes } \\
\text { Legionella Leptospira }\end{array}$ & $\begin{array}{l}\text { Mycobacterium } \\
\text { tuberculosis }\end{array}$ \\
\hline Fungi & $\begin{array}{l}\text { usually in } \\
\text { immunocompromised } \\
\text { individuals. }\end{array}$ \\
\hline Paryptococcus & $\begin{array}{l}\text { common in rural Australia but } \\
\text { usually found in } \\
\text { immunocompromised }\end{array}$ \\
\hline & $\begin{array}{l}\text { Malaria } \\
\text { Human African Trypanosomiasis (sleeping sickness) }\end{array}$ \\
\hline
\end{tabular}

*other viruses like West Nile, Japanese encephalitis and Louise encephalitis viruses are to be considered in specific geographical areas of the world.

The list of above microorganisms is not exhaustive and few common ones are provided

In South East Asia Japanese encephalitis virus is common whereas in Western Hemisphere the West Nile virus and St. Louise virus present particular problems. In immunocompromised individuals, most commonly those with Human Immunodeficiency Virus (HIV), and those on long term steroids and cytotoxic therapy, then other viruses become important in addition to those mentioned above, such as Epstein Barr Virus and Cytomegalovirus (CMV). Amongst the bacteria: Mycoplasma pneumonia and Mycobacterium tuberculosis are important with the latter more common in immunocompromised individuals. It is also important to state that fungi in the form of Cryptococcus neoformans should be looked for in all individuals when there is a high index of suspicion especially in sub-tropical Australia where it also affects immunocompetent individuals. With greater importance these days of rapid transcontinental air travel, parasites like Malaria, giving rise to cerebral malaria must not be missed as they are usually amenable to pharmacological therapy. In the case of Malaria delay in diagnosis and treatment has, in the past, proved disastrous for clinicians in temperate countries. Hence the value of accurate and comprehensive history and examination. In the end with ever changing epidemiology of arthropod-borne illnesses familiarity with local pattern of offending microorganisms is essential. Here the local department of microbiology presents a good resource of such information that would guide further investigations and treatment.

\section{Investigations}

Where there is a high index of suspicion of an individual presenting with infective encephalitis, then magnetic resonance imaging (MRI) of the head with gadolinium contrast be undertaken immediately

[2]. MRI head with contrast is necessary to show areas involved with inflammatory process as well as distinguishing encephalitic process from malignancy. Features to look for are diffuse white matter lesions and/or oedematous lobes of the brain or increased intensity of signals from the cortex. In some instances with early presentation of inflammatory process the MRI findings tend to be subtle and can easily be missed [3]. Figure 1 shows Flair image of MRI head of a patient in the forties who presented with acute onset of headache and pyrexia associated with confusion. Note the subtle oedematous changes as well as increased signals in subcortical areas in left parieto-occipital region. The patient made a good recovery after appropriate treatment. However, should 
facilities not be conducive to this imaging then computed tomography (CT) scan of the brain may be the initial investigation to rule out other causes of the presenting neurological dysfunction. This should lead to immediate lumbar puncture (LP), in the absence of contraindications, to get CSF for microscopy, protein estimation, culture for bacteria and PCR for the common viruses as described above and any other specific ones depending on the geographic location of the patient and presence of any immunodeficiency. In addition, request for serology (IgM) of the viruses and CSF IgM specific microorganism can be added to the investigations should PCR later prove to be negative.

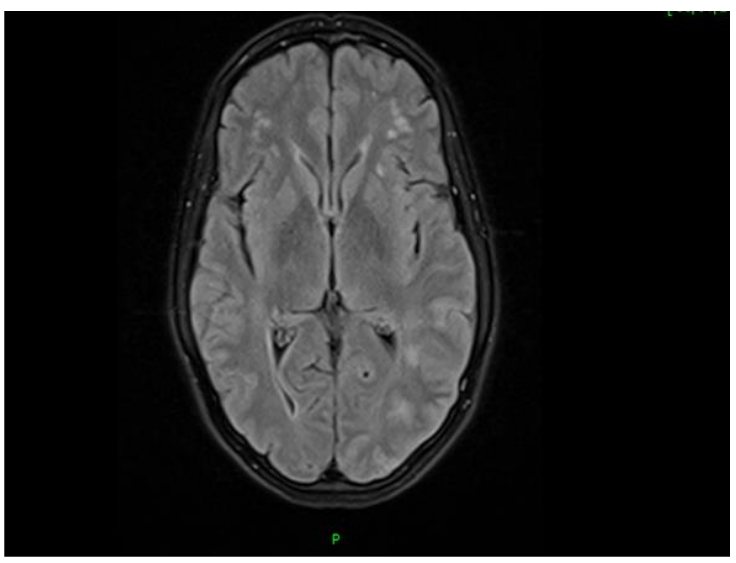

Figure 1: MRI head Flair image of patient with encephalitis. Note the swollen left parieto-occipital lobe compared to the right side.

In the event of low or absent lymphocyte count and protein concentration suggestive of absence of encephalitis, but the clinical picture strongly suggests it, then repeat LP is recommended 48 to 72 hours later when the inflammatory process has established itself and hence more reliable CSF findings tend to be present. One thing to stress is treatment should not be delayed in such situations and can be empirically undertaken as missing the diagnosis can be disastrous leading to increased mortality and morbidity in the form of memory impairment and inability to perform everyday tasks of personal care.

Additionally, blood/tissue cultures of other organs may need to be taken to identify bacterial organisms that may have resulted in secondary encephalitis such as Mycoplasma pneumonia or Tuberculosis. A chest $\mathrm{x}$-ray in these instances is useful. Other body fluids in the form of respiratory

secretions for viral PCR and relevant tissue biopsy, e.g. skin for Herpes simplex or zoster lesion, may also provide valuable clues to the diagnosis of the micro-organism involved. In instances of immunodeficiency then HIV serology would be required. It can be argued that HIV serology should be a mandatory part of the initial investigations in the evaluation of every patient with encephalitis as its presence tends to be pointer to other co-existing microorganisms which themselves may require effective treatment.

In instances of diagnostic difficulties despite the above investigative measures, an electroencephalogram (EEG) may prove useful in finally providing additional information required for the diagnosis of encephalitis, but this rarely necessary. By the same token, brain biopsy is an option in rare instances where there is still diagnostic difficulty and the patient is steadily worsening [4] but this tends to be undertaken in tertiary centres.

Despite the above modalities for diagnosing encephalitis, in significant cases the diagnosis is not confirmed early in the clinical presentation. However, in such cases where the clinical suspicion still remains because no other diagnosis could be entertained, with the passage of time such individuals get diagnosed with repeat appropriate imaging with MRI. Such a situation leads to delay in treatment and consequent neurological sequelae. There is thus, an urgent need of biomarkers either in the CSF and/or blood to provide 
complementary investigations in the early diagnosis of encephalitis whatever the aetiologic agent so that appropriate expeditious therapy is provided.

A study with 14 patients with infective encephalitis ( 8 with Japanese encephalitis, 1 Dengue and 5 non-specific encephalitis) [5] comparing with 10 controls found that CSF IL6 (Interleukin-6, a cytokine), RANTES (Regulated on Activation, Normal T Expressed and Secreted - chemokine) and IL-8 (chemokine) were increased in encephalitis. The extent of the rise of cytokines and chemokines did not correlate with severity of encephalitis or the radiological changes. These changes in IL- 6 and IL- 8 as well as RANTES were confirmed in another study involving Japanese encephalitis virus [6].

Similarly, in an article by Dafna BonnehBarkay [7], the usefulness of CSF IL- 6 in Herpes simplex encephalitis was also stressed with high levels found in those with poor clinical outcome. The article concludes that further study concerning the specificity of these interleukin biomarkers is required.

\section{Treatment}

There is limited choice of antimicrobial therapy for the wide variety of viruses causing encephalitis in adults. Herpes simplex Type I and II as well as Herpes zoster viruses contribute to significant numbers of aetiological agents causing encephalitis. They respond well to intravenous Acyclovir at $10 \mathrm{mg}$ per kilogram body weight eight hourly. Treatment with this antiviral drug must be started quickly as soon as there is a high index of suspicion coupled with the results of MRI head (or CT head) and CSF examination. This empirical approach to antimicrobial therapy is critical for the success of treatment in order to avoid increased mortality and morbidity $[8,9]$. The duration of treatment is 14 to 21 days depending on the clinical improvement observed and results of repeat lumbar puncture. MRI head findings in Herpes simplex virus are mainly confined to the temporal lobes but occasionally the inflammatory process may invade the contiguous part of the frontal lobe. Acyclovir has made an enormous impact on the survival of such patients, but despite early treatment some patients face long term morbidity in the form of neurological deficit, seizures and neuropsychological difficulties. In those who are immunocompromised where other organisms are responsible, CSF investigations can be hastened to provide answers while using empirically Acyclovir and Ceftriaxone (a broad spectrum antibiotic for bacterial infection). Obviously, those allergic to Penicillins, alternative antibiotics, in addition to Aciclovir, should be sought with advice from local microbiology department. There is no role for steroids in uncomplicated infective encephalitis.

Cytomegalovirus encephalitis is mainly an affliction of those who are immunocompromised but occasionally immunocompetent individuals are also affected [10]. MRI head with

gadolinium contrast shows features of ventriculitis with ependymal enhancement and CSF PCR coupled with serum and CSF CMV-specific IgM antibodies help establish the diagnosis. Treatment with gangciclovir is advocated for a period of 2 to 3 weeks and in those showing no clinical improvement then foscarnet should be tried $[1,11]$. It must be remembered that the outcome with CMV encephalitis is bleak without treatment leading to death in all cases and in those in whom treatment is started expeditiously more than half survive. The few immunocompetent individuals who succumb to this virus the outcome is good.

Mycoplasma pneumonia organism is known to cause pneumonia but it also 
produces encephalopathy and encephalitis $[12,13,14]$. Hence it is advisable in cases of encephalitis to undertake a chest $\mathrm{x}$-ray as part of the workup for such patients so that they get adequate and appropriate antimicrobial therapy very early, as discussed above.

The pathogenesis of HIV producing encephalitis is by the formation of multinucleated giant cells scattered in CNS [15]. However, the other important implication of such immunosuppression is the presence of opportunistic infections of CMV, Toxoplasma gondii and Cryptococcus neoformans. These organisms need to be looked for in the CSF with CSF PCR and specific antibodies, and when possible with serology, with treatment tailored to combating HIV infection and associated opportunistic infection [16].

Some other viruses causing encephalitis like West Nile Virus (now mainly in the Western Hemisphere), Japanese encephalitis virus (mainly in South East Asia) and St. Louise encephalitis virus (mainly in North America) which are spread by mosquitoes do not have specific antimicrobial therapy. The treatment is supportive and in some cases individuals have permanent neurological sequelae. Not surprisingly mortality tends to be high in those with severe disease. However, initial studies using intravenous immunoglobulin in Japanese virus encephalitis in endemic areas appear to be encouraging $[17,18]$, and this approach can be taken with other viruses where, as yet, no specific

antimicrobial therapy exists. Such an approach would provide another modality of treatment and may prove to be cheaper than specific antimicrobial therapy to be developed. Further studies in the use of intravenous immunoglobulins for other viruses, are therefore, urgently needed.

\section{Conclusion}

The diagnosis of infective encephalitis can be challenging. Imaging of the brain by MRI can be useful but sometimes in the early stages of the disease there can be subtle changes that can be missed and only identified retrospectively well after the diagnosis is made by other means. Therefore, there is an urgent need for getting easily available biochemical markers such as cytokines and chemokines that can rapidly help in the early diagnosis of various aetiological agents.

It cannot be stressed enough that early diagnosis is critical in providing effective treatment so as to avoid long term morbidity and mortality. Ultimately support from colleagues in Neurology and Infectious Diseases specialists may prove valuable and one should make use of this resource. By the same token there is an urgent need for newer specific viral therapies for those agents that at present, are not responsive to pharmacological therapy such as Enteroviruses, West Nile Virus, Japanese encephalitis and St.Louise encephalitis. The role of intravenous immunoglobulins in endemic areas where such viruses produce encephalitis may prove also be crucial in early specific therapy. This approach to treatment is just beginning to be realised with further studies required. Ultimately support from colleagues in Neurology and Infectious Diseases specialists may prove valuable and needs to be considered.

\section{References}

1) Trunkel AR, Glaser CA, Bloch $K C$ et al. The Management of Encephalitis: Clinical Practice Guidelines by the Infectious Diseases Society of America. Clinical Infectious Diseases 2008; 47: 303-327.

2) Kennedy PGE. Viral encephalitis: causes, differential diagnosis and management Journal of Neurology, Neurosurgery \& Psychiatry 2004; 75: i10-i15.

3) Ooi MH, Solomon T. Enteroviruses: Enterovirus 71. In : Kaslow RA, Stanberry LR, Le Duc JW, eds. Viral Infections of Humans. Epidemiology and Control. 5th ed. New York: Springer, 2014: 253-275. 
4) Solomon T, Michael BD, Smith PE et al. On behalf of the National Encephalitis Guidelines Development Group. Management of suspected viral encephalitis in adults: Association of British Neurologists and British Infection Association National Guideline. Journal of Infection 2012; 64: 347-373.

5) Kalita J, Srivastava R, Mishra MK et.al. Cytokines and chemokines in viral encephalitis: A clinicoradiological correlation. Neuroscience Letters 2010; 473: 48-51.

6) Winter PM, Dung NM, Loan HT. Proinflammatory Cytokines and Chemokines in Humans with Japanese Encephalitis. Journal of Infectious Diseases 2004; 190: 16181628.

7) Bonneh-Barkey D. Biomarkers of Encephalitis. In Hayasaka, D., Ed., In Tech. 2011. http://www.intechopen.com/books/pathogenesis-ofencephalitis/biomarkers-of-encephalitis.

8) Raschiles F, Wolff $M$, Delatour $F$ et al. Outcome of and Prognostic Factors for Herpes Simplex Encephalitis in Adult Patients: Results of Multicentre Study. Clinical Infectious Diseases 2002; 35: 254-260.

9) McGrath N, Anderson NE, Croxoson MC et al. Herpes simplex encephalitis treated with acyclovir: diagnosis and long term outcome', Journal of Neurology, Neurosurgery \& Psychiatry 1997; 63: 321-326.

10) Prosch S, Schielke E, Reip $A$ et al. Human Cytomegalovirus (HCMV) Encephalitis in Immnunocompetent Young Person and Diagnostic Reliability of HCMV DNA PCR Using Cerebrospinal Fluid of Nonimmunosuppressed Patients. Journal of Clinical Microbiology 1998; 36: 3636-3640.

11) Bamborschke $S$, Wullen $T$, Huber $M$ et al. Early diagnosis and successful treatment of acute cytomegalovirus encephalitis in a renal transplant recipient, Journal of Neurology 1992; 239 : 205-208.

12) Koskiniemi M. CNS manifestations associated with Mycoplasma pneumonia infections: summary of cases at the University of Helsinki and review. Clinical Infectious Diseases 1993; Suppl. 1: S52-S57.

13) Dionisio D, Valassina $M$, Mata $S$ et al. Encephalitis Caused Directly by Mycoplasma pneumonia. Scandinavian Journal of Infectious Diseases 1999; 31: 506-509.

14) Guleria R, Nisar N, Chawla TC et al. Mycoplasma pneumonia and central nervous complications: a review. Journal of Laboratory and Clinical Medicine 2005; 146: 55-63.

15) Wiestler OD, Leib SL, Brustle $O$ et al. Neurolopathology and pathologenesis of HIV encephalopathies. Acta Histochemica Supplement 1992; 42: 107-114.

16) Lang W, Miklossy J, Deruaz JP et al. Neuropathology of acquired immune deficiency syndrome (AIDS): a report of 135 consecutive autopsy cases from Switzerland. Acta Neuropathology 1989; 77: 79-390.

17) Caramello P, Canta F, Balbiano $R$ et al. Role of Intravenous Immunoglobulin Administration in Japanese Encephalitis. Clinical Infectious Diseases 2006; 43: 1620-1621.

18) Rayamajhi A, Nightingale $S$, Batta NK et al. Preliminary Double Blind Placebo-Controlled Trial of Intravenous Immunoglobulin for Japanese Encephalitis in Nepal. PLoS One 2015; 10(4): e0122608. 